\title{
On uniform recurrence of a direct product
}

\author{
Pavel V. Salimovلt" \\ Sobolev Institute of Mathematics, Novosibirsk, Russia. ch.cat.s.smi le@gmai l .com
}

received Sep 24, 2009, accepted June 1, 2010.

The direct product of two words is a naturally defined word on the alphabet of pairs of symbols. An infinite word is uniformly recurrent if each its subword occurs in it with bounded gaps. An infinite word is strongly recurrent if the direct product of it with each uniformly recurrent word is also uniformly recurrent. We prove that fixed points of the expanding binary symmetric morphisms are strongly recurrent. In particular, such is the Thue-Morse word.

Keywords: infinite words, uniform recurrence, strong recurrence, symmetric morphisms, Thue-Morse word

\section{Introduction}

Let $\Sigma=\Sigma_{s}=\{0,1, \ldots, s-1\}$ be a finite alphabet, and $x=x_{0} x_{1} \cdots \in \Sigma^{\mathbb{N}_{0}}$ be an infinite word on $\Sigma$ with indices in $\mathbb{N}_{0}=\mathbb{N} \cup\{0\}$.

A word $u$ is called a factor or a subword of a word $v=v_{0} v_{1} \ldots$ if $u=v_{i} v_{i+1} \ldots v_{i+n}$ for some $i$ and $n$. In this case we say the word $u$ occurs in $v$ at position $i$. A subword $u$ occurs in an infinite word $x$ uniformly if $u$ occurs in $x$ infinitely many times and the distances between contiguous positions where it occurs are bounded by some constant. An infinite word is uniformly recurrent if each its subword occurs in it uniformly.

The given definition of uniform recurrence is a paraphrase in terms of words of the original definition that came from works of Poincaré in classical mechanics and became the fundamental one of dynamics. The reason why it is studied from such a perspective is that the notion of recurrence turns out to be very useful in combinatorial number theory Furstenberg (1981). Applied to infinite words, it allows to prove new Ramsey type results and improve some classical ones like theorems of Hidman, van der Waerden and Szemeredi. At present time, uniform recurrence is a recognized property in factorial language theory, theoretical computer science and symbolic dynamics.

The direct product of two infinite words (or words of the same length) $x=x_{0} x_{1} \ldots$ on $\Sigma$ and $y=$ $y_{0} y_{1} \ldots$ on $\Delta$ is the word $x \otimes y=\left\langle x_{0}, y_{0}\right\rangle\left\langle x_{1}, y_{1}\right\rangle \ldots$ on the alphabet $\Sigma \times \Delta$. An infinite word $x$ is strongly recurrent if for each uniformly recurrent infinite word $y$ the product $x \otimes y$ also is uniformly recurrent. The property of strong recurrence is rare Furstenberg (1980) and not much is known on this subject. In the present paper we prove that words of some classical construction meet this property. More precisely, we speak of morphic sequences Allouche and Shallit (2003).

\footnotetext{
${ }^{\dagger}$ not supported.

1365-8050 @ 2010 Discrete Mathematics and Theoretical Computer Science (DMTCS), Nancy, France
} 
A morphism $\varphi: \Sigma^{*} \rightarrow \Delta^{*}$ is a mapping that obeys the identity $\varphi(x y)=\varphi(x) \varphi(y)$ for all words $x, y \in \Sigma^{*}$. We usually consider binary, i.e., $\Sigma_{2}^{*} \rightarrow \Sigma_{2}^{*}$ morphisms. A morphism $\varphi: \Sigma^{*} \rightarrow \Delta^{*}$ is an expanding one if for each $a \in \Sigma$ the inequality $|\varphi(a)|>1$ takes place.

A morphism is called uniform (m-uniform, where $m>1$ ) if the images of all letters are of the same length (equal to $m$ ). An $m$-uniform morphism $\varphi: \Sigma^{*} \rightarrow \Sigma^{*}$ is symmetric if for each $i \in \Sigma$ we have $\varphi(i)=\varphi(0) \oplus i^{m}$, where $\oplus$ denotes the symbol-by-symbol addition modulo $|\Sigma|$. Such a morphism is considered in Example 1 In the rest of the paper, when speaking of a symmetric morphism, we mean a binary symmetric morphism.

Any expanding morphism $\varphi: \Sigma^{*} \rightarrow \Sigma^{*}$ generates the mapping $\Sigma^{\mathbb{N}_{0}} \rightarrow \Sigma^{\mathbb{N}_{0}}$ that maps an infinite word $x=x_{0} x_{1} \ldots$ to the word $\varphi\left(x_{0}\right) \varphi\left(x_{1}\right) \ldots$ We denote it by the same letter $\varphi$ for convenience. When the word $\varphi(a)$ begins with $a$ for some $a \in \Sigma$, this mapping has fixed points, i.e., words satisfying $x=\varphi(x)$. These are exactly the words that can be obtained as $\operatorname{limits}_{\lim } \rightarrow \infty \varphi^{n}(a)=\varphi^{\omega}(a)$ for such $a \in \Sigma$.

Example 1 A classical morphic sequence is the Thue-Morse word $x_{T M}=01101001100101 \ldots$ that is a fixed point of the binary symmetric morphism $\varphi_{T M}$ defined by $\varphi_{T M}(0)=01$ and $\varphi_{T M}(1)=10$. This word can be obtained as a limit $\varphi_{T M}^{\omega}(0)$.

In this paper we prove that fixed points of the binary symmetric morphisms, in particular, the ThueMorse word are strongly recurrent.

\section{Words, languages and products}

The length of a word $u=u_{0} u_{1} \ldots u_{n-1}$ is $n$ and is denoted by $|u|$. We call a subword occurring in a word at position 0 a prefix of that word.

We use three equivalent Allouche and Shallit (2003) definitions of uniform recurrence.

Claim 1 For any infinite word $x$ the following statements are equivalent:

1. each subword of $x$ occurs in it uniformly

2. each prefix of $x$ occurs in it uniformly

3. for each integer $n$ there exists such $m$ that all subwords of $x$ of length $n$ occur in each subword of $x$ of length $m$.

For an infinite word $x$, let us define the recurrence function $R_{x}$ as the function that maps an integer $n$ to the smallest integer such that all subwords of $x$ of length $n$ occur in each subword of $x$ of length $R_{x}(n)$. This function is well-defined if and only if the word is uniformly recurrent. A subword $u$ of an infinite word $x$ is said to occur in $x$ non-uniformly if $x$ contains arbitrary long subwords where $u$ does not occur.

Let us denote the set of all finite words on $\Sigma$ by $\Sigma^{*}$, the set of all non-empty finite words by $\Sigma^{+}$and the set of all words of length $n$ by $\Sigma^{n}$.

A language is a subset of $\Sigma^{*}$. A language is factorial if it contains all subwords of its words. Let us denote the language of factors of a word $x$ by $F_{x}$ and the set of symbols occurring in $x$ by $\Sigma_{x}$. For a language $L$, let us denote by $\Omega_{L}$ the set of infinite words $x$ such that $F_{x} \subseteq L$. It is convenient to use the notation $\Omega_{x}$ instead of $\Omega_{F_{x}}$ for an infinite word $x$.

The cantor distance between infinite words $x$ and $y$ on $\Sigma$ is $d_{c}(x, y)=2^{-\inf \left\{i: x_{i} \neq y_{i}\right\}}$. Provided with such a distance the set $\Omega_{L}$ for an infinite factorial language $L \subseteq \Sigma^{*}$ is a compact metric space.

Let us give an example of the product of two uniformly recurrent words that is not uniformly recurrent. 
Example 2 Let the morphism $\varphi$ be the one defined by $\varphi(0)=011$ and $\varphi(1)=101$. Let us consider its fixed points $x=\varphi^{\omega}(0)=011101101101011101 \ldots$ and $y=\varphi^{\omega}(1)=101011101011101101 \ldots$ They are obviously uniformly recurrent but the word $x \otimes y$ is not.

Indeed, the word $x \otimes y$ is the fixed point beginning with $\langle 0,1\rangle$ of the morphism $\varphi^{\prime}$ defined by $\varphi^{\prime}(\langle a, b\rangle)=$ $\varphi(a) \otimes \varphi(b)$ for $a, b \in \Sigma_{2}$.

From the definition of $\varphi^{\prime}$ we see that there are arbitrary long subwords of $x \otimes y$ which wholly consist of symbols $\langle 0,0\rangle$ and $\langle 1,1\rangle$, hence the letter $\langle 0,1\rangle$ occurs in $x \otimes y$ non-uniformly.

A set $I \subseteq \mathbb{N}_{0}$ is called thick if it contains arbitrary long intervals (subsets of the form $\{a, a+1, \ldots, a+$ $n\}$ ). For example, if a subword occurs in an infinite word non-uniformly then the set of positions where it does not occur is thick.

Let $x=x_{0} x_{1} \ldots$ be an infinite word on $\Sigma$ and $I=\left\{i_{0}<i_{1}<\ldots\right\}$ be a subset of $\mathbb{N}_{0}$. By $x_{I}$ we denote the word $x_{i_{0}} x_{i_{1}} \ldots$ By $\left.x\right|_{I}$ we denote the restriction of the function $x: \mathbb{N}_{0} \rightarrow \Sigma$ to $I$.

Infinite words $x$ and $y$ are equivalent on the set $I \subseteq \mathbb{N}_{0}$ if there exists a bijection $h: \Sigma_{x_{I}} \leftrightarrow \Sigma_{y_{I}}$ such that $\left.h\left(\left.x\right|_{I}\right) \equiv y\right|_{I}$. In other words, $h$ is a renaming of symbols such that the equality $h\left(x_{i}\right)=y_{i}$ holds for each $i \in I$. In this case we use the notation $x \simeq_{I} y$. If these words are equivalent on the whole set $\mathbb{N}_{0}$, we use the notation $x \simeq y$ and call them just equivalent.

Claim 2 The direct product of strongly recurrent words is strongly recurrent.

Proof: Let infinite words $x$ and $y$ be strongly recurrent. The word $(x \otimes y) \otimes z \simeq x \otimes(y \otimes z)$ is uniformly recurrent for each uniformly recurrent word $z$. So $x \otimes y$ is strongly recurrent by the definition.

Let us define the block representation of order $m$ of an infinite word $x$ on an alphabet $\Sigma$ as the word $x^{[m]}=x_{0}^{[m]} x_{1}^{[m]} \ldots$ on $\Sigma^{m}$ such that $x_{i}^{[m]}=x_{i m} x_{i m+1} \ldots x_{i m+m-1}$.

An arithmetical subsequence of an infinite word $x$ is an infinite word of the form $x_{k} x_{k+d} x_{k+2 d} \ldots$ for arbitrary initial positions $k \geqslant 0$ and differences $d \geqslant 1$.

The following lemma belongs to the folklore, the proof can be found in Avgustinovich et al. (2003), for example.

Lemma 1 An arithmetical subsequence of a uniformly recurrent word is uniformly recurrent.

We use its corollary.

Corollary 1 A block representation of a word is uniformly recurrent if and only if the word is uniformly recurrent.

Proof: "If" part. Let an infinite word $x=x_{0} x_{1} \ldots$ on an alphabet $\Sigma$ be uniformly recurrent. Let us consider the word $x^{\prime}=x_{0}^{\prime} x_{1}^{\prime} \ldots$ on alphabet $\Sigma^{m}$ such that $x_{i}^{\prime}=x_{i} x_{i+1} \ldots x_{i+m-1}$. The word $x^{\prime}$ is uniformly recurrent since for its recurrence function we have $R_{x^{\prime}}(n)=R_{x}(n+m-1)$. The word $x^{[m]}=x_{0}^{\prime} x_{m}^{\prime} x_{2 m}^{\prime} \ldots$ is an arithmetical subsequence of $x^{\prime}$. Hence, by Lemma 4 it is uniformly recurrent.

"Only if" part. The word $x$ can be obtained from the word $x^{[m]}$ by applying the evident $m$-uniform morphism. The word $x$ is uniformly recurrent since for its recurrence function we have $R_{x}(n) \leqslant$ $R_{x^{[m]}}\left(\left\lceil\frac{n}{m}\right\rceil+1\right)$.

An infinite word $x=x_{0} x_{1} \ldots$ is periodic of a period $m$ if $x_{i}=x_{i+m}$ for each $i$.

Claim 3 Periodic words are strongly recurrent. 
Proof: Let $x$ be a periodic infinite word of a period $m$. Then for each uniformly recurrent word $y$ we have $(x \otimes y)^{[m]} \simeq y^{[m]}$. Using Corollary 4 of Lemma 4 we obtain that $x \otimes y$ is uniformly recurrent. So, $x$ is strongly recurrent.

The left shift operator denoted by $T$ maps an infinite word $x=x_{0} x_{1} \ldots$ to the word $T x=x_{1} x_{2} \ldots$ Note that if an infinite word $x$ is uniformly recurrent and $y \in \Omega_{x}$ then there exists an increasing progression $\left(n_{i}\right)_{i \in \mathbb{N}_{0}}$ such that $T^{n_{i}} x \rightarrow y$.

\section{Orbits of fixed points of binary symmetric morphisms}

In this section we investigate the equivalence on a set relation between the Thue-Morse or a similar word $x$ and words from $\Omega_{x}$. The result (Lemma 5 ) is that if $x \simeq_{I} y$ holds for a thick set $I \subseteq \mathbb{N}_{0}$, a fixed point $x$ of a binary symmetric morphism and a word $y \in \Omega_{x}$ then $x \simeq y$. This fact is a consequence of the circularity (recognizability) of such a words that is described further.

Let us call an infinite word $x(m, d)$-circular if for each its subword $u$ of length at least $d$ all positions of occurrences of $u$ in $x$ are equivalent modulo $m$. This is not the original definition introduced in Mignosi and Séébold (1993) and Cassaigne (1994) but the particular case of it. We call a fixed point of an $m$ uniform morphism circular if it is $(d, m)$-circular for some $d$.

Example 3 The Thue-Morse word $x_{T M}=01101001100101 \ldots$ is $(2,16)$-circular since each of its subwords of length at least 16 contains the subword 00 occurring in $x_{T M}$ only at odd positions.

The criterion for a fixed point of a morphism to be circular was formulated and proved in Frid (1998). A straightforward corollary of the result of that work is

Lemma 2 If a fixed point of an expanding binary symmetric morphism is not circular then it is periodic.

If an infinite word $x$ is periodic and $x \simeq_{I} y$ for some thick set $I \subseteq \mathbb{N}_{0}$ and $y \in \Omega_{x}$, then we obviously have $x \simeq y$. So, let us concentrate on circular words.

Claim 4 Let $x$ be an $(m, d)$-circular fixed point of a symmetric morphism $\varphi$ and $u, v \in F_{x}$. The next statements are true.

1. If $u$ is a prefix of $\varphi(v)$ and $|u| \geqslant d$ then $u$ occurs in $x$ only at positions divisible by $m$.

2. If $u \simeq v$ and $|u| \geqslant d$ then the positions of occurrences of $u$ and $v$ are equal modulo $m$.

Proof: In the conditions of the first statement we have $\varphi(v)$ occurring in $x$ at positions divisible by $m$, since $x=\varphi(x)$. Hence, the word $u$ occurs in $x$ at positions divisible by $m$ and only there, due to the definition of circularity.

For the second statement one may consider words $\varphi^{n}(0) \simeq \varphi^{n}(1)$ that occurs in $x$ at positions divisible by $m$. If $n$ is large enough, the word $u$ occurs in $\varphi^{n}(0)$ at some position $k$. Obviously, in this case $v$ occurs in $\varphi^{n}(0)$ or $\varphi^{n}(1)$ at the same position. Hence the statement is true due to the definition of circularity.

The basis of the main lemma is the following claim.

Claim 5 Let $x$ be a circular fixed point of an m-uniform symmetric morphism $\varphi$. Then for each $y \in \Omega_{x}$ there exist a unique word $z=z(y) \in \Omega_{x}$ and a unique number $t=t(y)<m$ such that $y=T^{t} \varphi(z)$. 
Proof: Let $x$ be $(m, d)$-circular. Then for the fixed point $x$ and an arbitrary integer $n$ we have $\varphi\left(T^{n} x\right)=$ $T^{m n} \varphi(x)=T^{m n} x$.

Let us consider a sequence $\left(n_{i}\right)_{i \in \mathbb{N}_{0}}$ of integers such that $T^{n_{i}} x \rightarrow y$. Since $x$ is circular, there are integers $i_{0}$ and $t<m$ such that for all $i \geqslant i_{0}$ we have $n_{i}=t \bmod m$. Hence, the sequence $\left(T^{\frac{n_{i}-t}{m}} x\right)_{i \geqslant i_{0}}$ of words is well-defined.

Due to the compactness of $\Sigma^{\mathbb{N}_{0}}$, there exists a subsequence $\left(T^{\frac{n_{i}-t}{m}} x\right)_{i \in I \subseteq \mathbb{N}_{0}}$ that converges to some word $z$. Obviously, $z \in \Omega_{x}$. For such a word $z$ we have

$$
T^{t} \varphi(z)=T^{t} \varphi\left(\lim _{i \in I} T^{\frac{n_{i}-t}{m}} x\right)=T^{t} \lim _{i \in I} \varphi\left(T^{\frac{n_{i}-t}{m}} x\right)=T^{t} \lim _{i \in I} T^{n_{i}-t} x=y .
$$

Suppose that some $z^{\prime} \in \Omega_{x}$ and $t^{\prime}<m$ satisfy $y=T^{t^{\prime}} \varphi\left(z^{\prime}\right)$.

Applying Claim 4 to prefixes of $\varphi(z)$ and $\varphi\left(z^{\prime}\right)$ we find that $y_{0} y_{1} \ldots y_{d-1}$ occurs in $x$ at positions equal to $t$ and $t^{\prime}$ modulo $m$. So, $t=t^{\prime}$ by the $d$-circularity of $x$.

The morphism $\varphi$ is symmetric hence $T^{t} \varphi(z)=T^{t} \varphi\left(z^{\prime}\right)$ implies $z=z^{\prime}$.

When $\sigma=\left(\sigma_{k}\right)_{k \in \mathbb{N}}$ is an infinite sequence of numbers satisfying $0 \leqslant \sigma_{k}<m$ for any $k$, let us denote by $S_{\sigma}(i)$ the sum $\sum_{j=1}^{i} m^{j-1} \sigma_{j}$. The statement of Claim 5 can be improved the following way.

Lemma 3 Let $x$ be a circular fixed point of an m-uniform symmetric morphism $\varphi$. Then for each $y \in \Omega_{x}$ there exist a unique sequence $\left(\sigma_{i}\right)_{i \in \mathbb{N}}$ of integers and a unique sequence $\left(y^{(i)}\right)_{i \in \mathbb{N}}$ of words, where $0 \leqslant$ $\sigma_{i}<m$ and $y^{(i)} \in \Omega_{x}$ for each $i$, such that

$$
y=T^{S_{\sigma}(i)} \varphi^{i}\left(y^{(i)}\right) \text { for every } i \in \mathbb{N} .
$$

Proof: Applying Claim 4 iteratively we can obtain sequences $\left(\sigma_{i}\right)_{i \in \mathbb{N}}$ of integers and $\left(y^{(i)}\right)_{i \in \mathbb{N}}$ of words, where $0 \leqslant \sigma_{i}<m$ and $y^{(i)} \in \Omega_{x}$ for each $i$, such that $y^{(i)}=T^{\sigma_{i+1}} \varphi\left(y^{(i+1)}\right)$ for each $i \in \mathbb{N}_{0}$ (supposing $y^{(0)}=y$ ). By the statement of Claim 4 , sequences satisfying such a property exist and are unique.

As it is easy to see

$$
\begin{aligned}
y= & T^{\sigma_{1}} \varphi\left(y^{(1)}\right)=T^{\sigma_{1}} \varphi\left(T^{\sigma_{2}} \varphi\left(y^{(2)}\right)\right)=T^{\sigma_{1}+m \sigma_{2}} \varphi^{2}\left(y^{(2)}\right)=\ldots \\
& \ldots=T^{S_{\sigma}(i)} \varphi^{i}\left(y^{(i)}\right)=\ldots
\end{aligned}
$$

We call such a sequence $\left(\sigma_{i}\right)_{i \in \mathbb{N}}$ the characteristic sequence of $y$ and denote it by $\sigma(y)$. We use this characteristic to prove that if a word $y \in \Omega_{x}$ is equivalent on a thick set to the fixed point $x$ of a binary symmetric morphism then $y$ is either $x$ itself or the another fixed point of this morphism.

The characteristic sequence of the fixed point itself is a sequence of 0 . One may expect the same from characteristic sequences of words that are equivalent on a thick set to the fixed point and it is true.

Lemma 4 Let $x$ be a circular fixed point of a symmetric morphism $\varphi$ and $y \in \Omega_{x}$. If $x \simeq_{I}$ y for a thick set $I \subseteq \mathbb{N}_{0}$ then $\sigma(y)$ consists only of 0 .

Proof: Let $x$ be $(m, d)$-circular. Suppose $\sigma_{1} \neq 0$. In this case $y=T^{\sigma_{1}} \varphi\left(y^{(1)}\right)$. 
The set $I$ is thick hence it contains the interval $B=\left\{m j+m-\sigma_{1}, m j+m-\sigma_{1}+1, \ldots, m j+m-\right.$ $\left.\sigma_{1}+d-1\right\}$ for some $j$. Let us consider the word $u=y_{B}$ that is equal to $x_{B}$. Due to the part 2 of Claim 4 the word $u$ occurs in $x$ only at positions equal to $m-\sigma_{1}$ modulo $m$.

On the other hand, we have $u=\varphi(v)$ where $v=y_{j+1}^{(1)} y_{j+2}^{(1)} \ldots y_{j+d}^{(1)}$ is a subword of $x$. Due to the part 1 of Claim 4, it implies that $u$ occurs in $x$ at positions divisible by $m$. This is the contradiction to the circularity of $x$.

Suppose now, that a number $i$ is the minimal satisfying $\sigma_{i} \neq 0$.

By Lemma 3 , there exists a unique word $y^{\prime} \in \Omega_{F_{x}}$ such that $y=T^{m^{i-1} \sigma_{i}} \varphi^{i}\left(y^{\prime}\right)=\varphi^{i-1}\left(T^{\sigma_{i}} \varphi\left(y^{\prime}\right)\right)$. For the fixed point $x$ we have $x=\varphi^{i-1}(x)$.

Since the morphism $\varphi$ is symmetric, the statement $\varphi^{i-1}\left(T^{\sigma_{i}} \varphi\left(y^{\prime}\right)\right) \simeq_{I} \varphi^{i-1}(x)$ implies the existence of a thick set $I^{\prime}$ such that $\left.T^{\sigma_{i}} \varphi\left(y^{\prime}\right)\right) \simeq_{I^{\prime}} x$. Doing the same reasoning as we did for the case $i=0$ we shall have the contradiction $\sigma_{i}=0$.

Now we are ready to prove the main lemma of this part. Using that equality of some word $y \in \Omega_{x}$ on a thick set to the fixed point $x$ implies that $\sigma(y) \equiv 0$, we will prove that equality on a thick set of some word to the fixed point implies equality of this words on the whole set $\mathbb{N}_{0}$.

Lemma 5 Let $x$ be a fixed point of a symmetric morphism and $y \in \Omega_{F_{x}}$. Then for each thick set $I \subseteq \mathbb{N}_{0}$ the statement $x \simeq y$ follows from $x \simeq_{I} y$.

Proof: If $x$ is periodic, the statement of the lemma is obviously true. So, due to Lemma2, we may restrict ourselves to the case $x$ being circular.

Lemma 4 states that in this case $\sigma(y)$ consists only of 0 . By Lemma 3 , for each $i$ there is a word $y^{(i)}$ satisfying $y=T^{S_{\sigma}(i)} \varphi^{i}\left(y^{(i)}\right)=\varphi^{i}\left(y^{(i)}\right)$. Hence the sequence $\left(\varphi^{i}\left(y_{0}^{(i)}\right)\right)_{i \in \mathbb{N}}$ of finite words is a sequence of growing prefixes of $y$ and $y=\lim _{i \rightarrow \infty} \varphi^{i}\left(y_{0}^{(i)}\right)$.

The morphism $\varphi$ is symmetric and has fixed points that implies $\varphi(a)_{0}=a$ for each $a \in \Sigma_{2}$. In view of this fact we have $y_{0}^{(i)}=y_{0}$ and $y=\lim _{i \rightarrow \infty} \varphi^{i}\left(y_{0}\right)$.

So, $y$ is either $x$ itself or another fixed point of $\varphi$ and therefore $x \simeq y$.

We conclude this section with the remark that a little bit more general statement than Lemma 5 can be proved using the same technique. It can be proved that if words $y, y^{\prime} \in \Omega_{x}$ are equivalent on a thick set $I$ for a fixed point $x$ of a binary symmetric morphism then there is an integer $t \in \mathbb{N}_{0}$ such that $T^{t} y \simeq T^{t} y^{\prime}$.

\section{Strong recurrence of fixed points of binary symmetric morphisms}

We start proving strong recurrence of fixed points of the binary symmetric morphisms with an observation of some case of not uniformly recurrent product.

Lemma 6 If the product $x \otimes y$ of uniformly recurrent words $x$ and $y$ is not uniformly recurrent and $x \simeq_{I} y$ for some thick set $I$, then there exists a word $x^{\prime} \in \Omega_{x}$ such that $x \otimes x^{\prime}$ is not uniformly recurrent and $x \simeq_{I} x^{\prime}$.

Proof: Let a bijection $h: \Sigma_{x_{I}} \leftrightarrow \Sigma_{y_{I}}$ be such that $h\left(x_{i}\right)=y_{i}$ for each $i \in I$.

The thick set $I$ contains arbitrary large intervals of $\mathbb{N}_{0}$, in particular, larger than $R_{x}(1)$ and $R_{y}(1)$. Therefore, for each symbol $a \in \Sigma_{x} \cup \Sigma_{y}$ there is an integer $i \in I$ such that either $x_{i}=a$ or $y_{i}=a$. So, $h$ 
is a bijection between whole alphabets $\Sigma_{x}$ and $\Sigma_{y}$ and the uniformly recurrent word $x^{\prime}=h^{-1}(y)$ is well defined. By the definition of $x^{\prime}$, we have $\left.\left.x^{\prime}\right|_{I} \equiv x\right|_{I}$.

Since the set $I$ is thick, it contains an interval $B=\left\{i, i+1, \ldots, i+R_{x^{\prime}}(n)-1\right\}$ for each $n$ and some $i=i(n)$. The word $x^{\prime}$ is uniformly recurrent thus each of its subwords of length $n$ occurs in the word $x_{B}^{\prime}$. Hence, the statement $x_{B}^{\prime}=x_{B}$ implies $F_{x^{\prime}} \cap \Sigma_{x}^{n} \subseteq F_{x} \cap \Sigma_{x}^{n}$. Therefore, $x^{\prime} \in \Omega_{x}$.

The product $x \otimes x^{\prime}$ is not uniformly recurrent because $x \otimes x^{\prime} \simeq x \otimes y$.

Now we came to the proof of the main result of this paper.

Theorem 1 Fixed points of the binary symmetric morphisms are strongly recurrent.

Proof: Let a word $x$ is the fixed point of an $m$-uniform symmetric morphism $\varphi$ and $y$ be a uniformly recurrent word. Without lose of the generality we may assume that $x=\varphi^{\omega}(0)$ and 1 occurs in $x$.

The idea of the proof is to show that if the product $x \otimes y$ is not uniformly recurrent then there exists a uniformly recurrent word $x^{\prime} \in \Omega_{x}$ such that the product $x \otimes x^{\prime}$ again is not uniformly recurrent and $x \simeq_{I} x^{\prime}$ for some thick set $I$. This is a contradiction to the statement of Lemma 5 .

Note that $x^{\left[m^{i}\right]} \simeq x$ for each $i$ since $x$ satisfy $x=\varphi^{i}(x)$ as a fixed point. The bijection between $\Sigma_{x_{\left[m^{i}\right]}}$ and $\Sigma_{x}$ that realizes the equivalence maps $\varphi^{i}(0)$ to 0 and $\varphi^{i}(1)$ to 1 .

Suppose that the product $x \otimes y$ is not uniformly recurrent and some prefix $p$ of $x \otimes y$ occurs in it non-uniformly. Let an integer $k$ satisfies $m^{k} \geqslant|p|$.

Consider the word $z=y_{\left[m^{k}\right]}$ that is uniformly recurrent by Corollary of Lemma .

Suppose that $(x \otimes z)_{i}=\left\langle 0, z_{0}\right\rangle$ for some $i$. Since $x \otimes z \simeq x^{\left[m^{k}\right]} \otimes z \simeq(x \otimes y)^{\left[m^{k}\right]}$, it implies that $p$ occurs in $x \otimes y$ at the position $i^{k}$. Thus the symbol $\left\langle 0, z_{0}\right\rangle$ occurs in $x \otimes z$ non-uniformly.

Now, let us consider the word $z^{\prime}=z^{\left[m^{l}\right]}$, where the integer $l$ satisfies $m^{l} \geqslant R_{z}(1)$. The word $z^{\prime}$ is uniformly recurrent by Corollary of Lemma .

Each symbol $u$ of $\Sigma_{z^{\prime}}$ is a word $u_{0} u_{1} \ldots u_{m^{l}-1}$ on $\Sigma_{z}$ that contains the symbol $z_{0} \in \Sigma_{z}$.

Let us define a morphism $\psi: \Sigma_{z^{\prime}}^{*} \rightarrow\{0,1\}^{*}$ by the following way. Let $\psi(u)=1$ if and only if the word $\varphi^{l}(0) \otimes u_{0} u_{1} \ldots u_{m^{l}-1}$ contains the symbol $\left\langle 0, z_{0}\right\rangle$. Otherwise, let $\psi(u)=0$ (in this case the symbol $\left\langle 0, z_{0}\right\rangle$ occurs in the word $\varphi^{l}(1) \otimes u_{0} u_{1} \ldots u_{m^{l}-1}$ since $\varphi$ is symmetric).

Let us consider the word $y^{\prime}=\psi\left(z^{\prime}\right)$ that obviously is uniformly recurrent.

Since the symbol $\left\langle 0, z_{0}\right\rangle$ occurs non-uniformly in the product $x \otimes z$, there are arbitrary large subwords of $x^{\left[m^{l}\right]} \otimes y^{\prime}$ that contains symbols $\left\langle\varphi^{l}(0), 0\right\rangle$ and $\left\langle\varphi^{l}(1), 1\right\rangle$ only. That implies the existence of a thick set $I$ such that $x^{\left[m^{l}\right]} \simeq_{I} y^{\prime}$. Using the fact $x^{\left[m^{l}\right]} \simeq x$ we obtain $x \simeq_{I} y^{\prime}$.

The word $x \otimes y^{\prime}$ is not uniformly recurrent since $x \otimes y^{\prime} \simeq x^{\left[m^{l}\right]} \otimes y^{\prime}$ and $x^{\left[m^{l}\right]} \otimes y^{\prime}$ contains arbitrary large subwords where the symbol $\left\langle\varphi^{l}(0), 1\right\rangle$ does not occur.

By Lemma 6 there exists a word $x^{\prime} \in \Omega_{x}$ satisfying to $x \simeq_{I} x^{\prime}$ for the thick set $I$ and the product $x \otimes x^{\prime}$ is not uniformly recurrent. On the other hand, we have $x \simeq x^{\prime}$ by Lemma 5 and hence $x \otimes x^{\prime}$ is uniformly recurrent. This is a contradiction we obtain supposing that $x \otimes y$ is not uniformly recurrent.

\section{Conclusion}

The problem of uniform recurrence of a direct product is still far from a solution.

We prove strong recurrence for the very particular classical case of infinite words. The same is true and will be done in subsequent papers for another classical constructions like Sturmian words (and some 
of its generalizations) and words constructed from strongly recurrent words by "Toeplitzation" method (including the Toeplitz words themselves). The problem is still open for interval exchange words that are another generalization in some sense of Sturmian words.

\section{Acknowledgements}

The author is grateful to Anna Frid and Sergey Avgustinovich for the useful discussions and to anonymous referees for the remarks that helped to essentially improve the readability of the paper. The author thanks the organization committee of AutoMathA 2009 for granting him the travel grant.

\section{References}

J.-P. Allouche and J. Shallit. Automatic Sequences. Theory, Applications, Generalizations. Cambridge University Press, Cambridge, 2003.

S. V. Avgustinovich, D. G. Fon-Der-Flaass, and A. E. Frid. Arithmetical complexity of infinite words. In M. Ito and T. Imaoka, editors, Words, Languages and Combinatorics III, pages 51-62. World Scientific Publishing, 2003.

J. Cassaigne. An algorithm to test if a given circular hd01-language avoids a pattern. In IFIP World Computer Congress'94, volume 1, pages 459-464. Elsevier, 1994.

A. Frid. On uniform dol words. In STACS'98, volume 1373 of LNCS, pages 544-554. Springer, 1998.

H. Furstenberg. Poincare recurrence and number theory. In The mathematical heritage of Henri Poincare, Proceedings of Symposia in Pure Mathematics, volume 39, pages 192-216. Amer. Math.Soc., 1980.

H. Furstenberg. Recurrence in Ergodic Theory and Combinatorial Number Theory. Princeton University Press, Princeton, 1981.

F. Mignosi and P. Séébold. If a d01 language is $k$-power-free then it is circular. In Automata, Languages and Programming, volume 700 of LNCS, pages 507-518. Springer, 1993. 